\title{
Determinants of hospitalization in Chinese patients with type 2 diabetes receiving a peer support intervention and JADE integrated care: the PEARL randomised controlled trial
}

Roseanne O. Yeung ${ }^{1,3,8^{*}}$, Jing-Heng Cai ${ }^{2}$, Yuying Zhang ${ }^{3,8}$, Andrea O. Luk ${ }^{3}$, Jun-Hao Pan², Junmei Yin ${ }^{3,8}$, Risa Ozaki ${ }^{3,8}$, Alice P. S. Kong ${ }^{3,8}$, Ronald Ma ${ }^{3,8}$, Wing-Yee So ${ }^{3,8}$, Chiu Chi Tsang ${ }^{4}$, K. P. Lau ${ }^{5}$, Edwin Fisher ${ }^{6}$, Williams Goggins ${ }^{3}$, Brian Oldenburg ${ }^{7}$ and Julianna Chan ${ }^{3,8}$

\begin{abstract}
Background: In a randomized controlled trial of 628 Chinese patients with type 2 diabetes receiving multidisciplinary care in the Joint Asia Diabetes Evaluation (JADE) Progam, 372 were randomized to receive additional telephone-based peer support (Peer Empowerment And Remote communication Linked by information technology, PEARL) intervention. After 12 months, all-cause hospitalization was reduced by half in the PEARL group especially in those with high Depression Anxiety and Stress Scale (DASS) scores.
\end{abstract}

Methods: We used stratified analyses, negative binomial regression, and structural equation modelling (SEM) to examine the inter-relationships between emotions, self-management, cardiometabolic risk factors, and hospitalization.

Results: Hospitalized patients were older, more likely to have heart or kidney disease, and negative emotions than those without hospitalization. Patients with high DASS score who did not receive peer support had the highest hospitalization rates. After adjustment for confounders, peer support reduced the frequency of hospitalizations by $48 \%$ with a relative risk of $0.52(95 \% \mathrm{Cl} 0.35-0.79 ; p=0.0018)$. Using SEM, improvement of negative emotions reduced treatment nonadherence (Est $=0.240, p=0.034$ ) and hospitalizations ( $E s t=-0.218, p=0.001)$. The latter was also reduced by an interactive term of peer support and chronic kidney disease (Est $=0.833, p=<0.001$ ) and that of peer support and heart disease ( $E s t=0.455, p=0.001$ ).

Conclusions: In type 2 diabetes, improvement of negative emotions and peer support reduced hospitalizations, especially in those with comorbidities, in part mediated through improving treatment nonadherence. Integrating peer support is feasible and adds value to multidisciplinary care, augmented by information technology, especially in patients with comorbidities.

Trial registration: NCT00950716 Registered July 31, 2009.

Keywords: Structural equation modelling, Quality improvement, Peer support, Integrated care, Hospitalizations, Negative emotions, Adherence

\footnotetext{
* Correspondence: ryeung@ualberta.ca

${ }^{1}$ Division of Endocrinology and Metabolism, Department of Medicine,

University of Alberta, 9-111K Clinical Science Building, 1135083 Avenue, Edmonton, AB T6G 2G3, Canada

${ }^{3}$ Chinese University of Hong Kong, Hong Kong, Hong Kong

Full list of author information is available at the end of the article
} 


\section{Background}

In clinical trial settings, diabetes complications are preventable with modifiable risk factor control. Despite recent advances in therapeutics, many patients have suboptimal control of cardiometabolic risk factors. In the United States, less than half of the patients have attained recommended goals for diabetes care [1]. In some surveys, patients with type 2 diabetes had 30\% increased risk of hospitalization [2] and $50 \%$ increased inpatient mortality rate compared to those without diabetes [3]. The co-occurrence of comorbidities, particularly poor mental health, also increases the risk of health care utilization in people with diabetes $[4,5]$.

One of the greatest challenges in diabetes management is to motivate, initiate, and sustain behavioural changes to maintain metabolic control [6]. While frequent contacts with health care providers (HCP) improves risk factor control [7], peer support has the potential to promote self-management and care quality, especially in low resource settings $[8,9]$. "Peer support" is defined as "support from a person who has experiential knowledge of a specific behaviour or stressor and similar characteristics as the target population" [8]. Herein, peer support can empower patients through building trust from shared experiences and by providing informational and emotional support [9].

In type 2 diabetes, peer support has been shown to improve clinical, behavioural, and psychological outcomes $[10,11]$. Peer support has also been shown to reduce health care utilisation and cost, although results have not always been consistent [12, 13]. In 2007, we launched a nurse-coordinated diabetes care program augmented by the web-based Joint Asia Diabetes Evaluation Program (JADE Program) [14]. We randomized 628 Chinese patients with type 2 diabetes receiving JADE care to peer support versus JADE alone to evaluate whether a telephone-based peer support program (Peer Empowerment And Remote communication Linked by information technology, PEARL program) would further improve risk factor control and all-cause hospitalizations [15]. At the end of 12 months, patients in both arms had similar improvements in glycemic control and cardiometabolic risk factors. However, the addition of PEARL peer support intervention reduced hospitalization rate compared to JADE care alone, especially in patients with the highest levels of negative emotion as measured by the Depression, Anxiety, and Stress Scale (DASS-21) with a relative risk (RR) of $0.15(0.07-$ $0.34, p<0.0001)$. In this post-hoc analysis, we performed detailed analysis to investigate the interrelationships between emotions, medication adherence, peer support, and hospitalizations.

\section{Methods}

\section{Study design and hypothesis}

The PEARL study was a 12-month randomized controlled trial evaluating the effect of peer support on metabolic control and hospitalizations from 2009 to 2010. The primary results of the PEARL study were published with detailed explanation of methods [15]. Our overarching hypothesis is that a telephone-based peer support intervention may improve negative emotions and medication adherence, thereby improving risk factor control and reducing all-cause hospitalization rates. To further explore the trial findings, we performed the following post-hoc analyses:

1) Univariate analyses of clinical profiles stratified by hospitalization status (ever hospitalized versus never hospitalized)

2) Univariate analyses of clinical profiles stratified by the median levels of negative emotions as measured by the Depression Anxiety Stress Scale (DASS-21) [15].

3) Kaplan Meier and negative binomial regression analyses to evaluate the impact of peer support and negative emotions (DASS-21) on hospitalizations.

4) Structural equation modelling (SEM), which provides a multivariate lens while addressing confounding by multicollinearity, to identify independent key factors leading to hospitalization.

\section{Ethics, consent, and permissions}

This study was approved by the Joint Chinese University of Hong Kong - New Territories East Cluster (CUHKNTEC) Clinical Research Ethics Committee. All willing patients gave written informed consent and completed validated questionnaires for evaluation of psychologicalbehavioural measures at baseline and at the end of the study, and were randomized to the PEARL peer support intervention within the JADE program or the JADE program alone in a 1:1 ratio.

\section{Setting}

Hong Kong has a subsidised public healthcare system with the Hospital Authority (HA) providing the bulk of chronic care through its hospitals and clinics which share a unified electronic medical record known as the Clinical Management System (CMS). Based on a series of epidemiological and interventional studies, the JADE Program was established in 2007 to deliver technologically-enhanced and integrated multidisciplinary care through the use of structured data collection, risk stratification, and evidence-based decision support [14].

This 12-month randomized study was conducted in three HA diabetes centres where the JADE Program was implemented. Consecutive patients were recruited throughout 
the year and randomized as they consented. The JADE Program included an initial 4-h nurse-led assessment including history taking, blood/urine collection, and eye/ft examination where the data were entered into the online portal which electronically generated personalised reports regarding the patient's risk factors, complications, and risk categories based on findings from the Hong Kong Diabetes Register [14]. Frequency of follow-up visits was recommended based on individualized risk assessment. Chronic kidney disease (CKD) was defined as estimated glomerular filtration rate $(\mathrm{eGFR})<60 \mathrm{ml} / \mathrm{min} / 1.73 \mathrm{~m}^{2}$ or dialysis-dependence. Heart disease was defined as a history of myocardial infarction, coronary revascularization, or heart failure.

\section{The PEARL intervention Peer supporters}

The details of the training program for peer supporters had been reported [15]. In brief, 33 motivated patients with HbA1c less than $8 \%(64 \mathrm{mmol} / \mathrm{mol})$ who had completed a 'Train the Trainer' program conducted during four 8-h workshops agreed to be peer supporters. The program focused on mindset, empathic listening, questioning skills, and counselling skills regarding lifestyle modification. Eligibility included Chinese adult patients with type 2 diabetes (18-70 years) who underwent the JADE comprehensive assessment. Exclusions included those in the lowest JADE Risk Category (no diabetes complications, $\leq 1$ risk factor, and low-risk score) [15] or those with suicidal ideation. Each peer supporter was introduced to 10 peers during a 2 -h session by nurses and was expected to contact each peer at least 12 times over 12 months in a phone or face-to-face meeting. Conversations or meetings were guided by a script, which addressed diet, exercise, self-monitoring of blood glucose, sick day management, foot care, emotional support, resources for information, and clinical care. Peer supporters were asked to record the duration of each call and any relevant details for the call. The doctors, nurses, and project coordinators met all peer supporters on three occasions for a half-day meeting to share experiences. Confidentiality of patient information was emphasised at each meeting.

\section{Peers}

All Chinese patients with type 2 diabetes aged 18 to 70 years who underwent JADE comprehensive assessments who were receptive were eligible. Exclusion criteria included illiteracy, inability to communicate in Chinese, those in JADE Risk Category 1 (no complications, $\leq 1$ risk factor, and low-risk score), and those receiving psychiatric treatment.

\section{Main outcomes and measures}

The CMS captured all hospitalizations within the Hong Kong public health care system, classified by the International Classification of Diseases, Ninth Revision (ICD9) codes on discharge. Measures included the total number of all-cause hospitalizations for each group, frequency of hospitalization per patient, and the total length of stay (TLOS) per patient admission, defined as a count of day admissions plus nights spent in a hospital. Measurements were censored using the CMS on the day of repeat assessment or after 12 months in those who did not return. All patients completed validated questionnaires for psychometric measures including the 9item Patient Health Questionnaire (PHQ-9) for depressive symptoms [16], the 21-item Depression Anxiety Stress Scale (DASS-21) with sub scores for depression, anxiety and stress [17], the 15-item Chinese Diabetes Distress Scale (CDDS-15) for diabetes-related distress [18], and the 4-item Morisky Medication Adherence Scale for medication adherence [19].

\section{Statistical analysis}

For each patient, we censored the hospitalization data at their 1 year reassessment. For patients who did not return at 1 year for assessment, we retrieved their results from the Health Authority CMS, if available. Descriptive analyses were presented as mean $\pm \mathrm{SD}$ or frequency (\%) stratified by hospitalization status and negative emotions. The Pearson $X^{2}$ test, Fisher exact test, $t$ test, Wilcoxon paired test, and Mann-Whitney test were used for comparisons, as appropriate. Significant negative emotion was defined as the top quintile value of DASS21 score $\geq 17$. We used unadjusted Kaplan Meier "time to event" estimates to illustrate the relationship between peer support and negative emotion on time to first hospitalization. Since the majority of patients were not hospitalized, we used zero-inflated negative binomial regression to estimate the relative risk (RR) for the frequency and TLOS of hospitalizations with 95\% confidence interval (CI) adjusted for age, sex, disease duration, and risk category to compare with the unadjusted findings. A $p<0.05$ (2-tailed) was considered significant. We applied intention-to-treat analysis to all randomized patients using the Statistical Package for Social Sciences (version 20.0, Chicago, USA) for descriptive analysis and Statistical Analysis Software for the negative binomial regression analysis (version 9.3). Missing data were omitted from the analyses.

\section{Structural equation modelling (SEM)}

We have chosen to use SEM because it allows examination of relationships among observed and latent (unobserved) variables. Moreover, SEM eliminates possible multicollinearity induced by highly correlated indicators 
and provides simple interpretations among observed and latent variables [20]. We first used factor analysis to group highly correlated measured variables into corresponding latent factors taking measurement errors into account. The structure of our factor loading was determined on subjective, a priori clinical knowledge. These included 1) "hospitalization" (grouped by TLOS and frequency of hospital admissions); 2) "change in negative emotions" from baseline to end of study (grouped by changes in CDDS-15, PHQ-9, and DASS-21); 3) "blood pressure" from systolic and diastolic blood pressure; 4) "lipids" from triglycerides, LDL-C, and total cholesterol; and 5) "obesity" from body mass index (BMI) and waist circumference. We did not include HbA1c due to similar changes between the two groups (PEARL: $-0.30(-0.47$ to -0.12$)$ and non-PEARL group: -0.29 ( -0.47 to 0.12 ) at one year [15]. In the SEM model, we included age, gender and peer support (yes/no: 1/0) as determinants for hospitalization based on results from our primary paper [15]. Supported by other studies, we included medication adherence (measured by the 4-item Morisky Medication Adherence Scale) [21] and diabetes complications [CKD and heart disease coded as 1/0)] known to increase health care utilization [22]. We regressed multiple equations linking peer support to "hospitalization" through "change in negative emotions", "adherence", "blood pressure", "lipids", and "obesity" factors (Fig. 1). We considered the effects of "change in negative emotions" on medication adherence and tested the interactive terms of (peer support $\times$ CKD) and (peer supportxheart disease) on "hospitalization". Mplus software was used to obtain the estimates and goodness-offit indices with optimal indexes defined as Chi square test $>0.05$, root mean square error of approximation smaller than 0.08 and Comparative Fit Index (CFI) value approximate to 1 [23].

\section{Results}

The study participants' clinical profiles have been published and were similar between the 2 groups [age:54.7 \pm 9.3 years; 57\% men; disease duration:9.4 \pm 7.7 years; HbAlc: $8.2 \% \pm$ $1.6 \%(66 \pm(-6) \mathrm{mmol} / \mathrm{mol}) ;$ systolic BP:136 $\pm 19 \mathrm{mmHg}$; LDL-C:2.89 $\pm 0.82 \mathrm{mmol} / \mathrm{L} ; 17.4 \%$ cardiovascular-renal disease; $34.9 \%$ insulin-treated] [15]. After a follow-up period of $414 \pm 55$ days, $144(22.9 \%)$ patients were hospitalized at least once. They were older, more likely to receive multiple medications, and 2-3 times more likely to have CKD and heart disease and had higher DASS-21 scores than those without hospitalization (Table 1). Those with the most negative emotions (DASS-21 $\geq 17, n=124$ ) were more likely to be female, less well-educated, more obese, had more depressive symptoms and poorer medication adherence than those with lower scores.

On Kaplan Meier analysis, patients who had DASS-21 $\geq$ 17 and did not receive peer support had the highest hospitalization rates than the other 3 groups stratified by

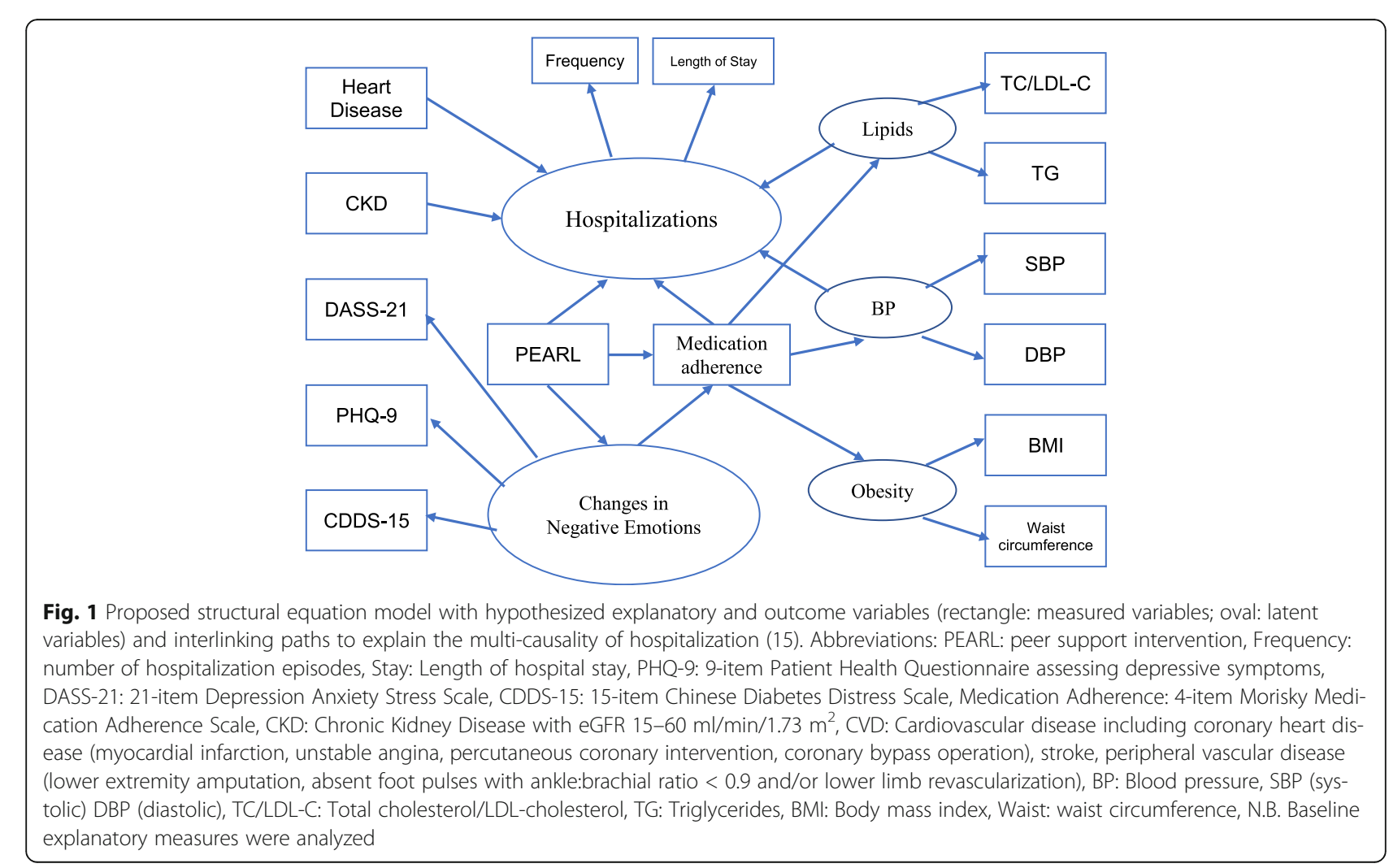


Table 1 Clinical, biochemical, psychological and behavioural parameters stratified by hospitalization and baseline DASS-21 score

\begin{tabular}{|c|c|c|c|c|c|c|}
\hline & \multicolumn{2}{|l|}{ Hospital admission } & \multirow[b]{2}{*}{$P$ value } & \multicolumn{2}{|l|}{ Baseline DASS-21 score } & \multirow[b]{2}{*}{$P$ value } \\
\hline & No $(n=484)$ & Yes $(n=144)$ & & $<17(n=503)$ & $\geq 17(n=124)$ & \\
\hline Age, years & $54 \cdot 1 \pm 9 \cdot 4$ & $56.7 \pm 8.6$ & 0.003 & $54.9 \pm 9.1$ & $54.0 \pm 9.7$ & 0.358 \\
\hline Male, n(\%) & $274(56 \cdot 6)$ & $81(56 \cdot 3)$ & 0.939 & $295(58 \cdot 6)$ & $59(47 \cdot 6)$ & 0.026 \\
\hline Diabetes duration, years & $9.1 \pm 7.7$ & $10 \cdot 3 \pm 7 \cdot 8$ & $0 \cdot 122$ & $9.4 \pm 7.7$ & $9.5 \pm 8.0$ & 0.845 \\
\hline \multicolumn{7}{|l|}{ Education } \\
\hline$<6$ years & $165(34 \cdot 1)$ & $63(43.8)$ & 0.079 & $171(34 \cdot 0)$ & $57(46 \cdot 0)$ & 0.005 \\
\hline $6-11$ years & $247(51 \cdot 0)$ & $62(43 \cdot 1)$ & & $252(50 \cdot 1)$ & $56(45 \cdot 2)$ & \\
\hline$>11$ years & $72(14.9)$ & $19(13 \cdot 2)$ & & $80(15.9)$ & $11(8.9)$ & \\
\hline \multicolumn{7}{|l|}{ Complications } \\
\hline Chronic kidney disease & $25(5 \cdot 2)$ & $21(14.8)$ & $<0.001$ & $34(6 \cdot 8)$ & $12(9 \cdot 8)$ & 0.251 \\
\hline Diabetic retinopathy & $166(34 \cdot 3)$ & $56(38.9)$ & 0.312 & $169(33 \cdot 6)$ & $53(42 \cdot 7)$ & 0.057 \\
\hline All heart events & $41(8.5)$ & $26(18 \cdot 3)$ & 0.001 & $52(10 \cdot 4)$ & $15(12 \cdot 1)$ & 0.586 \\
\hline \multicolumn{7}{|l|}{ JADE Risk categories } \\
\hline Very high risk & $73(15 \cdot 1)$ & $36(25 \cdot 0)$ & 0.003 & $86(17 \cdot 1)$ & $23(18.5)$ & 0.474 \\
\hline High risk & $385(79.5)$ & $104(72 \cdot 2)$ & & $391(77.7)$ & $97(78 \cdot 2)$ & \\
\hline Medium risk & $24(5 \cdot 0)$ & $4(2 \cdot 8)$ & & $24(4 \cdot 8)$ & $4(3 \cdot 2)$ & \\
\hline Low risk & $2(0.4)$ & $0(0 \cdot 0)$ & & $2(0.4)$ & $0(0 \cdot 0)$ & \\
\hline \multicolumn{7}{|l|}{ Treatments } \\
\hline Insulin & $165(34 \cdot 1)$ & $54(37.5)$ & 0.451 & $174(34 \cdot 6)$ & $45(36 \cdot 3)$ & 0.722 \\
\hline Oral anti-diabetic drugs & $407(84 \cdot 1)$ & $127(88 \cdot 2)$ & 0.226 & $423(84 \cdot 1)$ & $110(88 \cdot 7)$ & 0.197 \\
\hline On BP drugs & $305(63.0)$ & $104(72 \cdot 2)$ & 0.042 & $327(65 \cdot 0)$ & $81(65 \cdot 3)$ & 0.948 \\
\hline On lipid lowering drugs & $194(40 \cdot 1)$ & $84(58 \cdot 3)$ & $<0.001$ & $218(43 \cdot 3)$ & $59(47 \cdot 6)$ & 0.394 \\
\hline \multicolumn{7}{|l|}{ Risk factors control } \\
\hline Body mass index, $\mathrm{kg} / \mathrm{m}^{2}$ & $26 \cdot 8 \pm 4 \cdot 3$ & $27 \cdot 1 \pm 5 \cdot 0$ & 0.563 & $26 \cdot 6 \pm 4 \cdot 3$ & $27 \cdot 8 \pm 4 \cdot 8$ & 0.009 \\
\hline Systolic blood pressure, $\mathrm{mmHg}$ & $135 \cdot 6 \pm 18 \cdot 2$ & $136 \cdot 4 \pm 20 \cdot 9$ & 0.633 & $136 \cdot 2 \pm 18 \cdot 4$ & $134 \cdot 2 \pm 20 \cdot 7$ & 0.307 \\
\hline Diastolic blood pressure, $\mathrm{mmHg}$ & $80 \cdot 2 \pm 10 \cdot 5$ & $79 \cdot 0 \pm 11 \cdot 2$ & 0.227 & $80 \cdot 3 \pm 10 \cdot 5$ & $78 \cdot 6 \pm 11 \cdot 5$ & 0.115 \\
\hline Hemoglobin A1c, \% (mmol/mol) & $8.2 \pm 1.6(66.0 \pm 17.9)$ & $8.2 \pm 1.6(65.7 \pm 17.7)$ & 0.867 & $8.2 \pm 1.6(65.6 \pm 17.7)$ & $8.3 \pm 1.7(67.2 \pm 18.7)$ & 0.394 \\
\hline Total cholesterol, mmol/L & $4.91 \pm 1.07$ & $4.71 \pm 1.06$ & 0.044 & $4.83 \pm 1.07$ & $4.99 \pm 1.08$ & 0.160 \\
\hline Triglyceride, mmol/L & $1.40(1.00-2.00)$ & $1.49(1.00-1.97)$ & 0.704 & $1.40(1.00-1.97)$ & $1.52(1.00-2.30)$ & 0.076 \\
\hline $\mathrm{HDL}-\mathrm{C}, \mathrm{mmol} / \mathrm{L}$ & $1 \cdot 20 \pm 0.36$ & $1.23 \pm 0.36$ & 0.433 & $1 \cdot 21 \pm 0.35$ & $1 \cdot 17 \pm 0.37$ & 0.265 \\
\hline $\mathrm{LDL}-\mathrm{C}, \mathrm{mmol} / \mathrm{L}$ & $2 \cdot 92 \pm 0.78$ & $2 \cdot 77 \pm 0.91$ & 0.046 & $2 \cdot 86 \pm 0.79$ & $2.99 \pm 0.91$ & 0.131 \\
\hline Estimated GFR, $\mathrm{ml} / \mathrm{min} / 1 \cdot 73 \mathrm{~m}^{2}$ & $111.9 \pm 33.1$ & $102 \cdot 6 \pm 36 \cdot 8$ & 0.005 & $110 \cdot 2 \pm 33 \cdot 7$ & $107 \cdot 7 \pm 36 \cdot 1$ & 0.471 \\
\hline Microalbuminuria & $127(26 \cdot 6)$ & $39(27.5)$ & 0.843 & $134(27 \cdot 0)$ & $31(25 \cdot 6)$ & 0.765 \\
\hline \multicolumn{7}{|l|}{ Psychological assessment } \\
\hline PHQ-9 score & $4 \cdot 1 \pm 4 \cdot 1$ & $4.7 \pm 4.2$ & 0.131 & $3 \cdot 1 \pm 3 \cdot 1$ & $8 \cdot 6 \pm 4.9$ & $<0.001$ \\
\hline CDDS-15 score & $36 \cdot 8 \pm 13 \cdot 5$ & $37 \cdot 0 \pm 12 \cdot 2$ & 0.849 & $34 \cdot 8 \pm 12 \cdot 8$ & $45 \cdot 1 \pm 11 \cdot 6$ & $<0.001$ \\
\hline \multicolumn{7}{|l|}{ DASS-21 score } \\
\hline Total & $9 \cdot 3 \pm 10 \cdot 0$ & $14 \cdot 0 \pm 14 \cdot 0$ & $<0.001$ & $5 \cdot 9 \pm 4 \cdot 7$ & $28 \cdot 7 \pm 11 \cdot 3$ & $<0.001$ \\
\hline DASS-depression & $2.75 \pm 3.80$ & $4.23 \pm 5.02$ & 0.001 & $1.53 \pm 1.85$ & $9.48 \pm 4.78$ & $<0.001$ \\
\hline DASS-anxiety & $2.71 \pm 3.12$ & $4.20 \pm 4.63$ & $<0.001$ & $1.76 \pm 1.65$ & $8.33 \pm 4.39$ & $<0.001$ \\
\hline DASS-stress & $3.79 \pm 3.97$ & $5.24 \pm 5.02$ & 0.002 & $2.55 \pm 2.47$ & $10.59 \pm 4.01$ & $<0.001$ \\
\hline \multicolumn{7}{|l|}{ Medication adherence } \\
\hline High & $200(43 \cdot 8)$ & $65(47 \cdot 8)$ & $0 \cdot 248$ & $225(47 \cdot 3)$ & $40(34.5)$ & 0.021 \\
\hline
\end{tabular}


Table 1 Clinical, biochemical, psychological and behavioural parameters stratified by hospitalization and baseline DASS-21 score (Continued)

\begin{tabular}{|c|c|c|c|c|c|c|}
\hline & \multicolumn{2}{|c|}{ Hospital admission } & \multirow[b]{2}{*}{$P$ value } & \multicolumn{2}{|c|}{ Baseline DASS-21 score } & \multirow[b]{2}{*}{$P$ value } \\
\hline & No $(n=484)$ & Yes $(n=144)$ & & $<17(n=503)$ & $\geq 17(n=124)$ & \\
\hline Intermediate & $228(49 \cdot 9)$ & $66(48.5)$ & & $225(47 \cdot 3)$ & $68(58 \cdot 6)$ & \\
\hline Low & $29(6 \cdot 3)$ & $5(3 \cdot 7)$ & & $26(5 \cdot 5)$ & $8(6.9)$ & \\
\hline
\end{tabular}

Definitions of risk categories

1. Very High-Risk group with clinically evident cardiovascular-renal complications

2. High-Risk group with $\geq 3$ stratification parameters and/or values above the high specificity cut off for any one of the risk scores and/or eGFR< $60 \mathrm{ml} / \mathrm{min} / 1.73 \mathrm{~m}^{2}$

3. Medium Risk group with two stratification parameters and values above the high sensitivity cut off but lower than the high specificity cut off for any of the risk scores and eGFR between $60-90 \mathrm{ml} / \mathrm{min} / 1.73 \mathrm{~m}^{2}$ and

4. Low-Risk group with one or fewer stratification parameter and values below the high sensitivity cut off for all risk scores and eGFR $\geq 90 \mathrm{ml} / \mathrm{min} / 1.73 \mathrm{~m}{ }^{2}$

Definitions of complications and risk parameters:

Chronic kidney disease (CKD): estimated glomerular filtration rate $15-60 \mathrm{ml} / \mathrm{min}$ per $1.73 \mathrm{~m}^{2}$

Cardiovascular complication: coronary heart disease (myocardial infarction, unstable angina, percutaneous coronary intervention, coronary bypass operation), stroke, peripheral vascular disease (lower extremity amputation, absent foot pulses with ankle:brachial ratio $<0.9$ and/or lower limb revascularization)

Renal complications: End stage renal disease (ESRD) requiring dialysis and/or eGFR< $15 \mathrm{ml} / \mathrm{min} / 1.73 \mathrm{~m}^{2}$

Microalbuminuria: urinary albumin:creatinine ratio (ACR): $\geq 2.5 \mathrm{mg} / \mathrm{mmol}$ (men) and $3.5 \mathrm{mg} / \mathrm{mmol}$ (women)

Macroalbuminuria: urinary $A C R>25 \mathrm{mg} / \mathrm{mmol}$

Retinopathy (typical retinal changes including vitrectomy, haemorrhages, and exudates)

Medication adherence: measured by 4 -item Morisky Medication Adherence Scale where 4 is regarded as high adherence, score 2-3 as intermediate adherence, and $0-1$ as low adherence

peer support and DASS-21 scores (Fig. 2). Upon stratification by presence and absence of negative emotions at baseline, peer support tended to improve negative emotions reaching significance for medication adherence in those with the highest levels of negative emotions. Using negative binomial regression, and adjusting for age, gender, disease duration, JADE risk category, and DASS-21 score, peer support reduced hospitalization [RR 0.52 (95\% CI 0.35-0.79)] and shortened TLOS [RR 0.46 (95\% CI 0.25-0.85)].

\section{SEM findings}

Based on our original conceptual framework, we formulated a SEM that proposed explanatory pathways leading to hospitalization (Fig. 1). The "blood pressure", "lipids", or "obesity" pathways were not significant and thus were excluded from subsequent model building. After fitting data to the different pathways, the final SEM (Fig. 3) demonstrated a significant negative relationship of "change in negative emotions" on "hospitalization" and direct positive effect on "adherence". In other words, patients with reduced negative emotions were less likely to be hospitalized and were more adherent to medications. Since hospitalized patients were more likely to have CKD, heart disease, and negative emotions, we also tested the interactions between peer support and comorbidities on hospitalization and found strong positive interactions between peer support and CKD, as well as peer support and heart disease on hospitalization. Table 2 quantifies these relationships, which indicate that in patients without CKD, the difference in "hospitalization" values between the PEARL and nonPEARL group was -0.026 compared to $0.807(-0.026+$ $0.833=0.807)$ in those with CKD $(P=0.001)$. For heart disease, the respective values were -0.026 and 0.455 $(-0.026+0.455=0.429) \quad(P<0.001)$. Our model's goodness of fit indices revealed a Chi-squared test $p$-value of 0.1330 , indicating that our SEM model supported our hypothesis (i.e. not rejected by the data). The SEM model fitting was optimal with the root mean square error of approximation being 0.019 , and the CFI value being 0.978 , which were better than the predetermined respective criteria for model fitting (less than 0.08 and higher than 0.95).

\section{Discussion}

In our primary analysis of this 12-month randomized trial of 628 Chinese patients type 2 diabetes receiving JADEaugmented integrate care, cardio-metabolic risk factors improved in both groups with peer support reducing hospitalization rate, especially in those with negative emotions [15]. While peer support has been shown to improve glycemic control, few studies reported its impact on healthcare utilisation [24]. In this study, we used basic stratification analyses to identify differentiating characteristics between patients who had been hospitalized and those who had not. We then applied these characteristics in a SEM to account for the confounding effects of multicollinearity to better understand factors of hospitalization. We found a consistent, independent relationship between those with improvement in negative emotions and reduced hospitalization. We also found that patients with heart or kidney disease were more likely to have reductions in hospitalization when receiving peer support. Lastly, we found an independent relationship between those with improvement in negative emotions and improvement in medication adherence. 

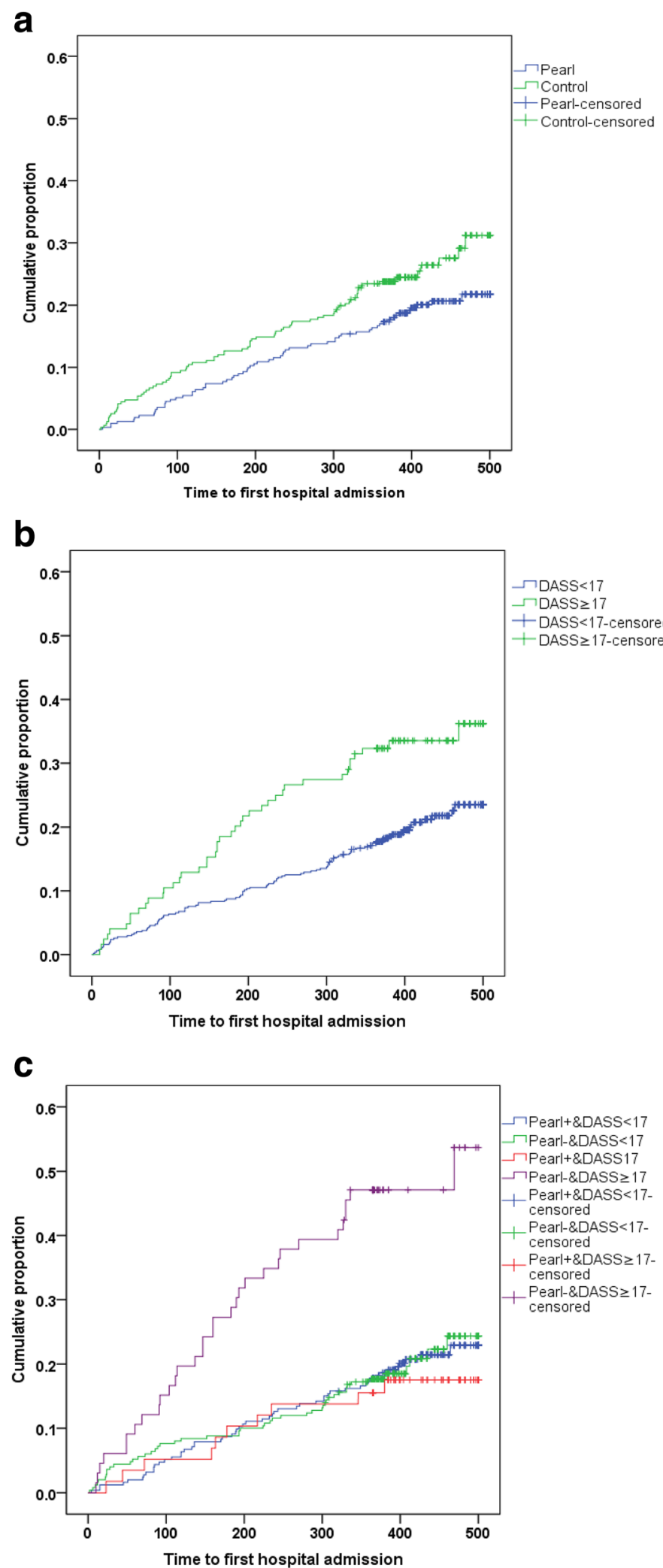

Fig. 2 (See legend on next page.) 
(See figure on previous page.)

Fig. 2 Kaplan Meier curves showing the cumulative proportions of people with type 2 diabetes requiring hospitalization. a Stratified by assignment to peer support (PEARL). b Stratified by baseline negative emotions (DASS $\geq 17$ ). c Stratified by peer support and baseline negative emotions. Pearl denotes peer support intervention. Depression Anxiety Stress Scale are denoted as DASS, using $\geq 17$ points as the cut off for those in the top quintile of subjects with negative emotions

\section{Peer support and its impact on comorbidity and hospitalization}

Our study suggests that those with more severe comorbidities may benefit more from peer support with regards to reduced hospitalization. In patients with CKD or CVD, peer support tended to improve psychometric, self-care, and adherence measures, albeit short of significance (data not shown). There is a paucity of research that has examined the use of peer support to reduce disease burden and health care utilization in patients with diabetes and CKD or CVD [25-27]. Our integrative analysis suggests that amongst all patients, patients with CKD and/or CVD might benefit the most from peer support to address their unmet needs.

\section{Improving negative emotions on hospitalization and the role of adherence}

Negative emotions can lead to increased health care utilisation in diabetes. In African Americans, the cooccurrence of diabetes and depression tripled the number of visits to emergency room and hospitalizations [28]. Other researchers have reported increased healthcare costs [29] and premature mortality [30] in people with diabetes and depression. Apart from depression, negative emotions such as anxiety, are more common in people with diabetes than healthy individuals [31]. Although anxiety in adolescents with diabetes is associated with a two-fold increase in hospital readmissions [32], similar findings have not been well documented in adult populations. Likewise, the roles of peer support and negative emotions in people with diabetes and their impact on health care utilisation have not been well-studied.

Our data show that improved negative emotions independently reduced hospitalizations and nonadherence, although we could not demonstrate a direct path between the latter two, likely due to small sample size. However, the independent effect of negative emotion on treatment adherence is noteworthy given the known impact of medication adherence on diabetes outcomes. Medication adherence independently predicted mortality and healthcare utilisation in a large British study of patients with type 2 diabetes receiving primary care [33]. In a survey of 2600 Chinese patients with type 2 diabetes, patients with depression and poor glycemic control reported frequent hypoglycemia, a common cause of hospitalization in diabetes, which was mediated in part by poor treatment adherence [34]. In a large study of insured Americans, improved medication adherence reduced the odds of hospitalization or visits to an emergency department by $13 \%$ after adjusting for comorbidities and socioeconomic status [35]. By converting a non-adherent to an adherent population, the authors modelled that close to a million hospitalizations and emergency department visits could be prevented in the United States.

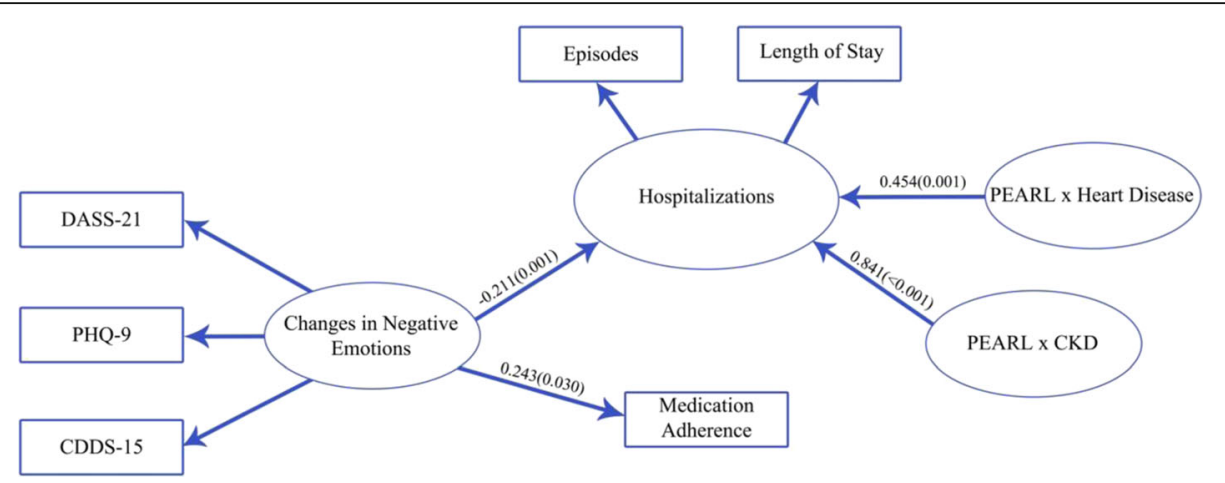

Fig. 3 A structural equation model (SEM) showing the effects of change in negative emotions (DASS, PHQ9, CDDS) on hospitalizations (admission episodes and stay) and medication adherence as well as the interaction between peer support and CKD/CVD on hospitalizations. Goodness of fit indices of the model: pvalue of the Chi-squared test is 0.1330; the root mean square error of approximation is 0.019 , the CFI value is 0.978 . The figure represents the estimates of latent variable relationships to outcome variables with pvalues in parentheses (refer to Table 2 for full details of the SEM). Abbreviations: PHQ-9: 9-item Patient Health Questionnaire assessing depressive symptoms, DASS-21: 21-item Depression Anxiety Stress Scale, CDDS-15: 15-item Chinese Diabetes Distress Scale, Medication Adherence: 4-item Morisky Medication Adherence Scale, CKD: Chronic Kidney Disease as estimated glomerular filtration rate 15-60 ml/min/1.73 m, CVD: Cardiovascular disease including coronary heart disease (myocardial infarction, unstable angina, percutaneous coronary intervention, coronary bypass operation), stroke, peripheral vascular disease (lower extremity amputation, absent foot pulses with ankle:brachial ratio $<0.9$ and/or lower limb revascularization) 
Table 2 Summary of the estimated coefficients of the measurement and structural equation

\begin{tabular}{|c|c|c|c|}
\hline \multicolumn{4}{|l|}{ Measurement equation } \\
\hline Latent variable & Indicators & Est. ( $P$ Value) & Standard Error \\
\hline \multirow[t]{3}{*}{ Change in negative emotions } & CDDS & 1.000 (ref) & NA (ref) \\
\hline & PHQ-9 & $0.874(<0.001)$ & 0.137 \\
\hline & DASS-21 & $1.390(<0.001)$ & 0.273 \\
\hline \multirow[t]{2}{*}{ Hospitalisation } & Length of stay & 1.000 (ref) & NA (ref) \\
\hline & Frequency of hospitalisation & $1.745(<0.001)$ & 0.098 \\
\hline \multicolumn{4}{|l|}{ Structural equation } \\
\hline Dependent (outcome) variable & Independent (explanatory) Variable & Est. ( $P$ Value) & Standard Error \\
\hline \multirow[t]{8}{*}{ Hospitalisation } & Change in negative emotions & $-0.218(0.001)$ & 0.065 \\
\hline & Medication adherence & $0.005(0.833)$ & 0.022 \\
\hline & Age & $0.013(0.552)$ & 0.022 \\
\hline & Peer support & $-0.026(0.576)$ & 0.046 \\
\hline & CKD & $0.065(0.580)$ & 0.118 \\
\hline & Heart disease & $-0.001(0.993)$ & 0.101 \\
\hline & Peer support×CKD & $0.833(<0.001)$ & 0.171 \\
\hline & Peer support $\times$ Heart disease & $0.455(0.001)$ & 0.141 \\
\hline Medication adherence & Change in negative emotions & $0.240(0.034)$ & 0.113 \\
\hline \multirow[t]{3}{*}{ Change in negative emotions } & Gender & $-0.022(0.684)$ & 0.054 \\
\hline & Education & $0.031(0.316)$ & 0.031 \\
\hline & Peer Support & $0.039(0.471)$ & 0.053 \\
\hline
\end{tabular}

Goodness of fit indices of the model: $p$-value of the Chi-squared test is 0.1330 ; the root mean square error of approximation is 0.019 , the CFI value is 0.978 CKD Chronic kidney disease defined by eGFR $<60 \mathrm{ml} / \mathrm{min} / 1.73 \mathrm{~m}^{2}$ or dialysis-dependence. Heart disease defined by a history of myocardial infarction, percutaneous coronary intervention, and heart failure

Although we did not show a significant interaction of peer support and negative emotions in our own SEM likely due to small sample size, the independent relationships of improved negative emotions on hospitalization reduction and improved medication adherence suggest the need for more research on these inter-relationships.

\section{Strengths and limitations}

Strengths of this study include the collection of thorough physical, psychological and behavioural assessments in conjunction with documentation of healthcare utilisation, which are important quality measures. We also provide a detailed description of a technologically-enhanced integrated care and peer support program within a real-world setting. Regarding limitations, we found that some of the hypothesized paths, for example, effects of peer support on hospitalization through negative emotions and adherence, were not supported by the final model, which might be due to the strong confounding effect of JADE care through comprehensive assessment and education in all patients. Psychometric measurements were only measured between pre and post intervention, so changes in negative emotions could be modulated by hospitalization itself, thereby confounding the effect. We also recognized that the metabolic parameters (BP, obesity, lipids) resulted in poor fit in the SEM analysis, although univariate analysis showed that patients with or without hospitalizations had similar control of cardiometabolic risk factors. Furthermore, the greater effect size of peer support on daytime than nighttime admissions were not immediately evident. It is plausible that peer support could only alleviate hospitalizations arising from minor events which might be linked to negative emotions, but not those due to serious illness. Despite these limitations, the factor loadings, path coefficients, and model statistics of our final SEM were significant, supporting our conclusions on the relationships between peer support, comorbidities, medication adherence, and hospitalization. That said, due to the exploratory nature of this post hoc analysis and the relatively small sample size, the positive effects of a peer support intervention on hospitalization require replication and more detailed investigations.

In chronic diseases, factors other than negative emotions and medication adherence, such as ethnic minority status and incomes can also influence clinical outcomes. These parameters were not evaluated in our study. We also did not measure and adjust for comorbidities unrelated to diabetes or capture private health care utilisation. However, these unmeasured variables were expected to be equally distributed 
between the two groups given adequate randomization. The generalizability of this study was limited by the volunteer bias, as only $23 \%$ of eligible patients agreed to be peer supported, suggesting a more targeted approach is needed in implementing these peer support programs.

\section{Conclusion}

In patients with type 2 diabetes receiving technologicallyenhanced multidisciplinary care, reduction in negative emotions reduced hospitalization and medication nonadherence, while peer support reduced hospitalization in patients with cardiovascular-renal complications. Taken together, these findings highlight the complex inter-relationships between physical and psychological health and hospitalization, and the importance of structuring care processes so as to identify high-risk patients who might benefit from additional peer support.

\begin{abstract}
Abbreviations
CDDS-15: 15-item Chinese Diabetes Distress Scale; CKD: Chronic kidney disease as estimated glomerular filtration rate $15-60 \mathrm{ml} / \mathrm{min} / 1.73 \mathrm{~m}^{2}$; CVD: Cardiovascular complication including coronary heart disease (myocardial infarction, unstable angina, percutaneous coronary intervention, coronary bypass operation), stroke, peripheral vascular disease (lower extremity amputation, absent foot pulses with ankle:brachial ratio $<0.9$ and lower limb revascularization); DASS-21: 21-item Depression Anxiety Stress Scale; JADE: Joint Asia Diabetes Evaluation; Morisky Medication Adherence Scale: 4-item Medication Adherence Score; PEARL: Peer Empowerment And Remote communication Linked by information technology; PHQ-9: 9-item Patient Health Questionnaire assessing depressive symptoms
\end{abstract}

\section{Acknowledgements}

We thank all participants, physicians, nurses, and support staff in making the project possible. Special thanks to all the peer supporters, Vanessa Lau, Renee Tse, Angela Pang, Yee Mui Lee, Cindy Lui, and all staff at the Asia Diabetes Foundation and Yau Chung Kit Diabetes Assessment Centre in coordinating the peer support program.

\section{Funding}

The study was supported by the American Academy of Family Physicians Foundation Peers for Progress Program through the Eli Lilly and Company Foundation awarded to the Asia Diabetes Foundation (ADF). The ADF was supported through an unrestricted Merck educational grant. ROY received funding support from the University of British Columbia Clinical Investigator Program for a 2-year fellowship at the CUHK and ADF. JHC and JHP received funding support from the National Natural Science Foundation of China (NSFC $11101443,11271383,11301555)$. The funders did not have any role in the study design, data collection or analysis or preparation of the manuscript. JCNC is the guarantor and takes full responsibility for the work as a whole, including the study design, access to data, and the decision to submit and publish the manuscript.

\section{Availability of data and materials}

The datasets generated and analyzed during the current study are not publicly available because consent was not obtained for sharing of data outside of the research institution. Data is stored on the servers of the Asia Diabetes Foundation in Hong Kong SAR.

\section{Authors' contributions}

Study concept and design: JCNC, BO, EF, AOL, JHC, ROY, JY, YZ. Acquisition, analysis, or interpretation of data: All authors. Drafting of the manuscript: ROY, JCNC, JHC, BO, EF, AOL, APSK, YZ. Critical revision of the manuscript for important intellectual content: All authors. Statistical analysis: JHC, YZ, WG, JP, JCNC, JY, ROY. Obtained funding: JCNC, EF, BO. Administrative, technical, or material support: JCNC, JHC, BO, WS, RO, EF, YZ, ROY. All authors read and approved the final manuscript.

\section{Ethics approval and consent to participate}

This study was approved by the Joint CUHK-NTEC Clinical Research Ethics Committee. Agreeable patients gave written informed consent. This trial was pre-registered at clinicaltrial.gov under identifier NCT00950716 Registered July 31, 2009.

\section{Consent for publication}

Consent for publication of raw data was not obtained and the dataset could in theory, pose a threat to confidentiality. The researchers did not specifically ask for anonymized data to be deposited in the public repository. All data are anonymized and captured using the portal implemented through the Asia Diabetes Foundation established as a knowledge transfer project in the form of a registered charitable research organization governed by the CUHK Foundation. A patient log was kept by the clinics for identification and linkage to the hospitalization data using the CMS governed by the Hong Kong Hospital Authority, which is the main public healthcare provider in Hong Kong.

\section{Competing interests}

JCNC has received research grant and honorarium for consultancy or giving lectures from Eli Lilly. All research grants were awarded to the CUHK or ADF. All honoraria have been donated to the Chinese University of Hong Kong to support diabetes research and education. JCNC is also the Chief Executive Officer (pro bono) of ADF, a charitable organisation governed by the CUHK Foundation (www.adf.org.hk) which develops the web-based JADE program to conduct quality improvement and translational research programs through private-public partnerships. EF is the Global Director of the Peers for Progress, supported by the American Academy of Family Physicians Foundation and funded by Eli Lilly Company and Foundation. Other authors declared no conflict of interest relevant to this manuscript.

\section{Publisher's Note}

Springer Nature remains neutral with regard to jurisdictional claims in published maps and institutional affiliations.

\section{Author details}

'Division of Endocrinology and Metabolism, Department of Medicine, University of Alberta, 9-111K Clinical Science Building, 1135083 Avenue, Edmonton, AB T6G 2G3, Canada. ² un Yat-Sen University, Guangzhou, China. ${ }^{3}$ Chinese University of Hong Kong, Hong Kong, Hong Kong. ${ }^{4}$ Alice Ho Miu Ling Nethersole Hospital, Hong Kong, Hong Kong. ${ }^{5}$ North District Hospital, Sheung Shui, Hong Kong. ' University of North Carolina at Chapel Hill, Chapel Hill, USA. ${ }^{7}$ University of Melbourne, Melbourne, Australia. ${ }^{8}$ Asia Diabetes Foundation, Hong Kong, Hong Kong.

Received: 31 July 2017 Accepted: 20 February 2018

/ Published online: 07 March 2018

\section{References}

1. Ali MK, Bullard KM, Saaddine JB, Cowie CC, Imperatore G, Gregg EW. Achievement of goals in U.S. diabetes care, 1999-2010. N Engl J Med. 2013; 368:1613-24. https://doi.org/10.1056/NEJMsa1213829.

2. Bo S, Ciccone G, Grassi G, Gancia R, Rosato R, Merletti F, et al. Patients with type 2 diabetes had higher rates of hospitalization than the general population. J Clin Epidemiol. 2004;57:1196-201. https:// doi.org/10.1016/j.jclinepi.2004.02.015.

3. Umpierrez GE, Isaacs SD, Bazargan N, You X, Thaler LM, Kitabchi AE. Hyperglycemia: an independent marker of in-hospital mortality in patients with undiagnosed diabetes. J Clin Endocrinol Metab. 2002;87:978-82.

4. Calderón-Larrañaga A, Abad-Díez JM, Gimeno-Feliu LA, Marta-Moreno J, González-Rubio F, Clerencia-Sierra M, et al. Global health care use by patients with type-2 diabetes: does the type of comorbidity matter? Eur J Intern Med. 2015;26:203-10. https://doi.org/10.1016/j.ejim.2015.02.011.

5. Khalid JM, Raluy-Callado M, Curtis BH, Boye KS, Maguire A, Reaney M. Rates and risk of hospitalisation among patients with type 2 diabetes: retrospective cohort study using the UK general practice research database linked to English hospital episode statistics. Int J Clin Pract. 2013:1-9. https://doi.org/10.1111/ijcp.12265.

6. Ahola AJ, Groop PH. Barriers to self-management of diabetes. Diabet Med. 2013;30:413-20. https://doi.org/10.1111/dme.12105. 
7. Norris SLS, Lau J, Smith S, Smith SJ, Schmid C, Engelgau M. Selfmanagement education for adults with type 2 diabetes a meta-analysis of the effect on glycemic control. Diabetes Care. 2002;25:1159-71. http://care. diabetesjournals.org/content/25/7/1159.short. Accessed 12 Feb 2014

8. Dennis CL. Peer support within a health care context: a concept analysis. Int J Nurs Stud. 2003;40:321-32. https://doi.org/10.1016/S0020-7489(02)00092-5.

9. Heisler M. Ovenview of peer support models to improve diabetes self management and clinical outcomes. Diabetes Spectr. 2007;20:214-21. http:// spectrum.diabetesjournals.org/content/20/4/214.short. Accessed 10 Feb 2014

10. Keyserling TC, Samuel-Hodge CD, Ammerman AS, Ainsworth BE, HenríquezRoldán CF, Elasy TA, et al. A randomized trial of an intervention to improve self-care behaviors of African-American women with type 2 diabetes: impact on physical activity. Diabetes Care. 2002;25:1576-83. http://www. ncbi.nlm.nih.gov/pubmed/12196430. Accessed 10 Feb 2014

11. Lorig K, Ritter PL, Villa FJ, Armas J. Community-based peer-led diabetes self-management: a randomized trial. Diabetes Educ. 2009;35:641-51. https://doi.org/10.1177/0145721709335006

12. Gillespie P, O'Shea E, Paul G, O'Dowd T, Smith SM. Cost effectiveness of peer support for type 2 diabetes. Int J Technol Assess Health Care. 2012;28: 3-11. https://doi.org/10.1017/S0266462311000663.

13. Pennington $M$, Visram $S$, Donaldson C, White M, Lhussier M, Deane $K$, et al. Cost-effectiveness of health-related lifestyle advice delivered by peer or lay advisors: synthesis of evidence from a systematic review. Cost Eff Resour Alloc. 2013;11:30. https://doi.org/10.1186/1478-7547-11-30.

14. Chan JCN, Ozaki R, Luk A, Kong APS, Ma RCW, Chow FCC, et al. Delivery of integrated diabetes care using logistics and information technology-the joint Asia diabetes evaluation (JADE) program. Diabetes Res Clin Pract. 2014; 106(Suppl):S295-304. https://doi.org/10.1016/S0168-8227(14)70733-8.

15. Chan JCN, Sui Y, Oldenburg B, Zhang Y, Chung HHY, Goggins W, et al. Effects of telephone-based peer support in patients with type 2 diabetes mellitus receiving integrated care. JAMA Intern Med. 2014;174:972-81. https://doi.org/10.1001/jamainternmed.2014.655.

16. Kroenke K, Spitzer RL, Williams JB. The PHQ-9: validity of a brief depression severity measure. J Gen Intern Med. 2001;16:606-13.

17. Lovibond PF, Lovibond SH. The structure of negative emotional states: comparison of the depression anxiety stress scales (DASS) with the Beck depression and anxiety inventories. Behav Res Ther. 1995;33:335-43.

18. Ting RZ, Nan H, Yu MW, Kong AP, Ma RC, Wong RY, et al. Diabetes-related distress and physical and psychological health in chinese type 2 diabetic patients. Diabetes Care. 2011;34:1094-6. https://doi.org/10.2337/dc10-1612.

19. Morisky DE, Green LW, Levine DM. Concurrent and predictive validity of a self-reported measure of medication adherence. Med Care. 1986:24:67-74

20. Bollen KA. Structural equations with latent variables. 1989. http:// www.loc.gov/catdir/bios/wiley041/88027272.html.

21. Sokol MC, KA MG, Verbrugge RR, Epstein RS. Impact of medication adherence on hospitalization risk and healthcare cost. Med Care. 2005;43: 521-30. http://www.ncbi.nlm.nih.gov/pubmed/15908846

22. Karahalios A, Somarajah G, Hamblin PS, Karunajeewa H, Janus ED. Quantifying the hidden healthcare cost of diabetes mellitus in Australian hospital patients. Intern Med J. 2017; https://doi.org/10.1111/imj.13685.

23. Muthén L, Muthén B. Mplus user's guide (version 7.0). Muthén and Muthén; 2007. https://www.statmodel.com/download/usersguide/ Mplus\%20user\%20guide\%20Ver_7_r3_web.pdf.

24. Dale JR, Williams SM, Bowyer V. What is the effect of peer support on diabetes outcomes in adults? A systematic review. Diabet Med. 2012;29: 1361-77. https://doi.org/10.1111/j.1464-5491.2012.03749.x.

25. Bello AK, Qarni B, Samimi A, Okel J, Chatterley T, Okpechi IG, et al. Effectiveness of multifaceted care approach on adverse clinical outcomes in nondiabetic CKD: a systematic review and meta-analysis. Kidney Int reports. 2017;2:617-25. https://doi.org/10.1016/j.ekir.2017.02.007.

26. Wan TTH, Terry A, Cobb E, McKee B, Tregerman R, Barbaro SDS. Strategies to modify the risk of heart failure readmission: a systematic review and meta-analysis. Heal Serv Res Manag Epidemiol. 2017;4: 2333392817701050. https://doi.org/10.1177/2333392817701050

27. Taylor F, Gutteridge R, Willis C. Peer support for CKD patients and carers: overcoming barriers and facilitating access. Health Expect. 2016;19:617-30. https://doi.org/10.1111/hex.12348.

28. Husaini BA, Hull PC, Sherkat DE, Emerson JS, Overton MT, Craun C, et al. Diabetes, depression, and healthcare utilization among African Americans in primary care. $J$ Natl Med Assoc. 2004;96:476-84. http://www.pubmedcentral.nih.gov/ articlerender.fcgi?artid=2595010\&tool=pmcentrez\&rendertype $=$ abstract
29. Hutter N, Schnurr A, Baumeister $\mathrm{H}$. Healthcare costs in patients with diabetes mellitus and comorbid mental disorders-a systematic review. Diabetologia. 2010;53:2470-9. https://doi.org/10.1007/s00125-010-1873-y.

30. Sullivan $M, O^{\prime}$ Connor $P$, Feeney $P$, Hire D, Simmons DL, Raisch D, et al. Depression predicts all-cause mortality. Diabetes Care. 2012;35:1708-15.

31. Rubin RR, Peyrot M. Psychological issues and treatments for people with diabetes. J Clin Psychol. 2001;57:457-78. https://doi.org/10.1002/jclp.1041.

32. Garrison MM, Katon W, Richardson L. The impact of psychiatric comorbidities on readmissions for diabetes in youth. Diabetes Care. 2005;28:2150-4.

33. Currie CJ, Peyrot M, Morgan CL, Poole CD, Jenkins-Jones S, Rubin RR, et al. The impact of treatment noncompliance on mortality in people with type 2 diabetes. Diabetes Care. 2012;35:1279-84. https://doi.org/10.2337/dc11-1277.

34. Zhang Y, Ting RZ, Yang W, Jia W, Li W, Ji L, et al. Depression in Chinese patients with type 2 diabetes: associations with hyperglycemia, hypoglycemia, and poor treatment adherence. J Diabetes. 2015:"n/a-n/a. doi: https://doi.org/10.1111/1753-0407.12238.

35. Jha AK, Aubert RE, Yao J, Teagarden JR, Epstein RS. Greater adherence to diabetes drugs is linked to less hospital use and could save nearly $\$ 5$ billion annually. Health Aff (Millwood). 2012;31:1836-46. https://doi.org/10.1377/hlthaff.2011.1198.

\section{Submit your next manuscript to BioMed Central and we will help you at every step:}

- We accept pre-submission inquiries

- Our selector tool helps you to find the most relevant journal

- We provide round the clock customer support

- Convenient online submission

- Thorough peer review

- Inclusion in PubMed and all major indexing services

- Maximum visibility for your research

Submit your manuscript at www.biomedcentral.com/submit
) Biomed Central 